PAPER

\title{
Internal jugular vein valve incompetence and intracranial venous anatomy in transient global amnesia
}

\author{
S J Schreiber, F Doepp, R Klingebiel, J M Valdueza
}

J Neurol Neurosurg Psychiatry 2005;76:509-513. doi: 10.1136/jnnp.2004.043844

See end of article for authors' affiliations

Correspondence to:

Stephan J Schreiber,

Department of Neurology,

University Hospital

Charité, Schumannstr. 20/

21, 10117 Berlin,

Germany; Stephan.

Schreiber@charite.de

Received 20 April 2004

Revised version received

6 July 2004

Accepted 6 August 2004
Background: Recently a causal relation between internal jugular vein valve incompetence (IJVVI) and transient global amnesia (TGA) has been suggested. IJVVI is postulated to provoke a transient mesiotemporal ischaemia by venous congestion. This mechanism requires a patent venous pathway from the affected IJV through the transverse sinus, confluens, straight sinus (SS), vein of Galen into the basal vein of Rosenthal and the internal cerebral veins.

Objective: To study IJVVI in TGA patients in relation to the intracranial venous anatomy.

Methods: IJVVI was defined if a repeated Valsalva manoeuvre (VM) led to a retrograde jugular flow detected by extracranial duplex ultrasound. Non-contrast venous MR angiography (MRA) was performed to analyse intracranial drainage patterns of the SS in relation to the side of the IJVVI. SS drainage was differentiated into three groups: predominantly right, left, and bilateral drainage. Ultrasound studies were performed in 25 TGA patients and 85 age matched controls. Twenty patients underwent venous MRA. Results: Sixty eight per cent of patients and 33\% of controls showed unilateral or bilateral IJVVI ( $p=0.0025$ ). In $36 \%$ of patients a TGA preceding VM was reported. Drainage pattern of SS and side of IJVVI corresponded in five of eight patients $(63 \%)$ with VM and four of 12 patients without VM $133 \%$, $\mathrm{p}=0.0994)$.

Conclusion: Our study confirms the significantly higher prevalence of IJVVI in TGA patients. However, no specific IJVVI related intracranial venous drainage patterns could be found to further support the hypothesis of a direct causal relation between IJVVI and TGA.
$\mathrm{T}$ ransient global amnesia (TGA) is a benign condition characterised by sudden anterograde amnesia of less than 24 hours without any further focal neurological deficit. It predominantly affects people older than 50 years and reoccurs in about $3-4 \%$ of cases only. In up to $10 \%$, the amnesia may be accompanied by headaches, dizziness, nausea/vomiting, and neck pain. ${ }^{12}$ Reported preceding factors range from emotional and physical stress, sexual intercourse, severe pains, sudden rapid temperature changes, and coughing attacks to iatrogenic triggering by cerebral angiography. ${ }^{3-5}$ However, the underlying pathomechanism remains unclear. Several hypotheses, including arterial transient ischaemic attacks, epileptic seizures, hyperventilation, migraine, and spreading depression have been postulated and controversially discussed. ${ }^{356}$ Recently, a transient mesiotemporal ischaemia induced by venous congestion was suggested as a further potential TGA cause. ${ }^{7}$ The postulated mechanism is thought to occur through a Valsalva-like manoeuvre triggered by some of the above mentioned preceding activities. Supporting evidence for this hypothesis was contributed by three ultrasound studies, which found a significantly higher prevalence of retrograde internal jugular vein (IJV) blood flow-attributed to IJV valve incompetence (IJVVI)—during a Valsalva manoeuvre (VM) in TGA patients compared with controls. ${ }^{8-10}$ However, a mesiotemporal venous congestion implies a sufficiently patent venous pathway from the affected IJV through the sigmoid sinus, transverse sinus, confluens, the unpaired straight sinus (SS), and vein of Galen into the paired basal vein of Rosenthal and internal cerebral veins, which drain the mesiotemporal brain regions (fig 1). The SS collects blood from both sides but often delivers it predominantly unilateral into one transverse sinus. The present study analyses IJVVI in TGA patients in relation to the SS drainage side by means of extracranial duplex ultrasound and intracranial venous magnetic resonance angiography (MRA).

\section{METHODS}

Study group

Patients were prospectively enrolled into the study if they fulfilled the Hodges criteria for TGA. ${ }^{3}$ Controls were recruited from routine outpatients sent to our ultrasound laboratory if they were free of any relevant arterial atherosclerotic disease-that is, free of haemodynamically relevant extracranial or intracranial stenoses (grade of stenosis $<50 \%$ ). Exclusion criteria for either group were a decompensated congestive heart failure or a history of central venous IJV cannulation. All patients and controls gave informed consent.

\section{Ultrasound protocol}

Duplex ultrasound was performed in patients and controls in a head straight, supine position with a 7-11 MHz linear transducer (Powervision 6000, SSA-370A, Toshiba, Tokyo, Japan). IJV b-mode image and Doppler flow were continuously insonated approximately $2 \mathrm{~cm}$ above the internal jugular valves using the triplex mode of the ultrasound machine. The Doppler sample volume was set between 0.5 and $1 \mathrm{~cm}$ and placed in the centre of the IJV lumen. IJVVI was assessed on both sides after repeated training of the

Abbreviations: IJV, internal jugular vein; IJVVI, internal jugular vein valve incompetence; MIP, maximum intensity projections; MRA, magnetic resonance angiography; SS, straight sinus; TGA, transient global amnesia; TOF, time-of-flight; VM, Valsalva manoeuvre 




Figure 1 Schematic drawing of the venous drainage pathways for the mesiotemporal region (1). The blood drains through the paired basal vein of Rosenthal and internal cerebral veins $(2+3)$ into the unpaired vein of Galen and the straight sinus $(4+5)$. The straight sinus may drain unilaterally into one or equally into both transverse sinus (7) and from there through the sigmoid sinus (8) into the internal jugular vein (9). (6) Confluens sinuum. (Figure adapted from Feneis H. Anatomische Bildnomenklatur. 2nd edition. Stuttgart: Thieme Verlag, 1970).

subjects during three maximal VMs (duration $>5 \mathrm{~s}$ ). Pictures of the VM were stored on magneto-optical disk. A sufficient VM was confirmed by a clearly visible increase of IJV diameter (approximately $1 \mathrm{~cm}$ or more) in the b-mode image. IJVVI was concluded if retrograde jugular flow could be seen in the Doppler spectrum during each VM (fig 2). Reduction of flow velocity or zero flow was rated as competent jugular valves.

\section{MRI protocol}

Intracranial non-contrast venous MRA was performed in patients only using $1.5 \mathrm{~T}$ scanners (Magnetom Vision or
Magentom Symphony, Siemens, Erlangen, Germany). For data acquisition a two dimensional time-of-flight (TOF) MRA protocol was applied (TR $30 \mathrm{~ms}$, TE $6.5 \mathrm{~ms}$, FA $50^{\circ}, 3 \mathrm{~mm}$ slice thickness, I excitation $7.19 \mathrm{~min}$, $250 \mathrm{~mm}$ FOV, matrix $256 \mathrm{~mm}$ ). Off-line analysis of the three dimensional MRA data sets was carried out subsequent to data transfer through the intranet on two workstations (SIENET MagicView VB33/Siemens, Germany, and Vitrea/Vital images, USA) using maximum intensity projections (MIP) of about $4 \mathrm{~mm}$ slab thickness orientated along the SS axis (fig 3). The SS, confluens, and adjacent horizontal parts of both TS were included in the

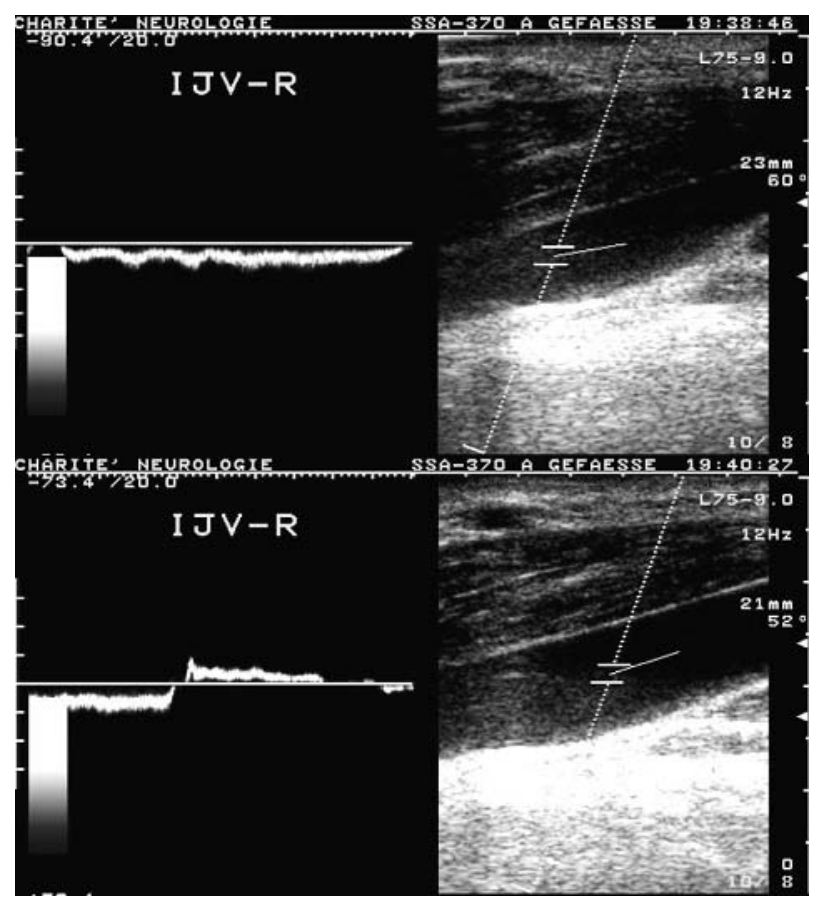

Figure 2 Triplex mode ultrasound images of a patient with internal jugular vein valve incompetence in the right jugular vein. Top: resting condition. Doppler spectrum analysis reveals an orthograde continuous flow in the internal jugular vein (IJV). Bottom: image under Valsalva manoeuvre. Note the increased diameter of the IJV (b-mode image, right side) and the temporary reversal of blood flow in the Doppler spectrum. 

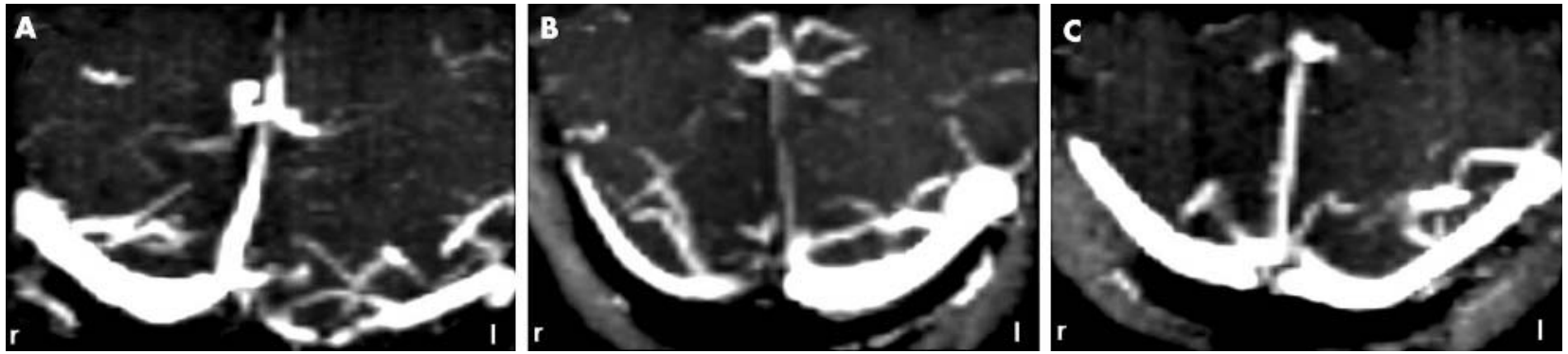

Figure 3 Venous MR angiography images demonstrating different straight sinus (SS) drainage types. A: right sided SS drainage, B: left sided SS drainage, C: bilateral SS drainage. r, right side; l, left side.

slab volume. Appearance of the SS drainage side was evaluated by two investigators (RK, SJS) unaware of the presence or side of IJVVI and differentiated into three groups: a predominantly right sided (group A), a predominantly left sided (group B), and a bilateral drainage (group C, fig 3).

\section{Statistical analysis}

All presented values are given as means \pm standard deviation. Two-sided Fisher's exact test was performed for comparison of controls and patients. A p value $<0.05$ was considered significant.

\section{RESULTS}

\section{Clinical findings}

Twenty six patients were screened; one had to be excluded because of intracranial bilateral high grade stenoses within the carotis-t region. Twenty five patients (9 women, 16 men) aged $61 \pm 10$ years (range: $37-77$ years) and 85 controls ( 37 women, 48 men) aged $62 \pm 9$ years (range 48-81 years) were included. The average duration of amnesia in patients was $7 \pm 5$ hours (range 20 minutes to 18 hours). Nine of 25 patients (36\%) had a reliable history of a probable preceding VM (house cleaning and moving of furniture, football play, car mending, fitting a carpet, carrying of flower pots, digging in the garden, sawing wood, skiing, cough attack).

\section{IJV valve incompetence}

Ultrasound analysis for IJVVI was performed and evaluated in all controls and patients. IJVVI was found in 28 (33\%) controls and in $17(68 \%)$ of TGA patients yielding a significant difference $(\mathrm{p}=0.0025)$. Table 1 illustrates the distribution of right sided, left sided, and bilateral IJVVI, which did not differ between both groups. Two of the eight patients with competent valves $(25 \%)$ and seven of the 17 with IJVVI $(41 \%, \mathrm{p}=0.66)$ had a preceding VM.

\section{Venous MRA}

MRA was performed in 20 of 25 TGA patients. Analysis of SS anatomy revealed a predominantly right sided drainage in eight $(40 \%)$, a left sided drainage in five $(25 \%)$, and a bilateral drainage in seven $(35 \%)$ cases. Thirteen of the 20 patients did have a IJVVI and eight a preceding VM. The relation of SS drainage and IJVVI sides of patients with and without VM is illustrated in Table 2. IJVVI and SS drainage corresponded in five $(63 \%)$ of the eight patients with VM and four $(33 \%)$ of the 12 patients without a reported VM $(\mathrm{p}=0.0994)$.

\section{DISCUSSION}

The venous congestion hypothesis for TGA, originally postulated by Lewis in $1998,^{7}$ is primarily based on the theoretical consideration that the onset of symptoms often correlates with prior Valsalva-like activities, which may lead to venous reflux through the IJV. IJVVI may be caused by valve insufficiency or aplasia. In anatomical studies, aplasia has been reported to occur in up to $16 \%$ of non-selected patients whereas valve competence was not assessed. ${ }^{11}$ Analysing IJV valve function, three US studies found a significantly higher prevalence of IJVVI in TGA patients, which supports the theory of a causal relation. Sander et al analysed the extent of duplex colour inversion during VM whereas Akkawi et al ${ }^{10}$ used an echo-contrast method evaluating the number of refluxing contrast bubbles in the IJV. Both research groups reported an IJVVI prevalence between 70 and $73 \%$ in patients compared with 30 to $47 \%$ in controls. $^{8-10}$ Data of the current study (68\% and 33\%) confirm this phenomenon and also indirectly validate our chosen duplex US protocol. In addition, the distribution of the affected sides in patients and controls (right/left/bilateral: 68/ $32 / 0 \%$ and $59 / 35 / 6 \%$ ) appears to be similar to data from Akkawi et al $(60 / 3 / 37 \%$ and $89 / 0 / 11 \%) .{ }^{10}$ As the high prevalence of right sided IJVVI might correspond to the frequently observed right sided anatomical predominance of IJV drainage, ${ }^{12-14}$ we additionally performed jugular blood volume flow measurements, which did not yield a correlation between IJV flow dominance and the side of the IJVVI (data not shown).

Assuming a causal and not coincidental relation of IJVVI and TGA, two further preconditions must be accomplished to effectively induce a mesiotemporal blood flow disturbance.

Table 1 Number and distribution of internal jugular vein valve incompetence (IJVVI) in 85 controls and 25 patients

\begin{tabular}{lcc}
\hline & Controls & Patients \\
\hline Total IJVVI & $28(100 \%)$ & $17(100 \%)$ \\
Right sided IJVVI & $19(68 \%)$ & $10(59 \%)$ \\
Left sided IJVI & $9(32 \%)$ & $6(35 \%)$ \\
Bilateral IJVVI & - & $1(6 \%)$
\end{tabular}


Table 2 Distribution of jugular vein valve incompetence (IJVVI) in 20 patients in relation to straight sinus (SS) drainage side (group $\mathrm{A}-\mathrm{C}$ ) and preceding Valsalva manoeuvre (VM)

\begin{tabular}{|c|c|c|c|c|c|c|}
\hline & \multicolumn{2}{|c|}{ Group A } & \multicolumn{2}{|c|}{ Group B } & \multicolumn{2}{|c|}{ Group C } \\
\hline & All & With VM & $\overline{\text { All }}$ & With VM & $\overline{\text { All }}$ & With VM \\
\hline Right sided IJVVI & 3 & 1 & 1 & 1 & 5 & 3 \\
\hline Left sided IJVVI & 3 & - & 1 & 1 & - & - \\
\hline Competent valves & 2 & - & 3 & 1 & 2 & 1 \\
\hline
\end{tabular}

Group A: right sided SS drainage; group B: left sided SS drainage; group C: bilateral SS drainage. Bold numbers indicate plausible constellations according to the hypothesis (left: matching SS drainage and IJVVI; right: matching SS drainage and IJVVI, including a reported VM).

These are a patent venous pathway and a plausible history of a VM. Concerning the intracranial venous anatomy, the unpaired SS, collecting blood from both mesiotemporal regions through the basal veins of Rosenthal and internal cerebral veins, is a key vessel as its drainage pattern into the confluens can vary considerably. ${ }^{14}{ }^{15}$ Interestingly, the correspondence of predominant SS drainage and IJVVI sides was slightly but not significantly higher in patients with a VM (63\%) compared with those without (33\%, $p=0.0994)$. However, these findings still leave the TGA in $37 \%$ of cases with VM unexplained by the hypothesis. Finally, we could not see any further specific venous morphological characteristics in our TGA patients. Unfortunately, these findings do not deliver further evidence in favour of the venous congestion hypothesis. Yet, they also do not reliably exclude the postulated mechanism as some methodological limitations of our study have to be considered: venous MRA is an increasingly used technique for diagnosis or exclusion of sinus venous thrombosis. ${ }^{16}$ However, non-contrast MRA in regions with complex flow patterns might lead to MR signal loss and misinterpretation of venous anatomical variants. ${ }^{17}$ Furthermore, the side of the predominant SS drainage might not be as relevant as postulated because intravascular pressure changes can also be transmitted through a non-dominant small or hypoplastic but communicating vessel segment. Also, the postulated functional disturbance might not require a specific morphological correlate as various other pathways of the complex valveless intracranial venous network-for example, the vertebral veins or the paraspinal venous plexus could as well transmit the venous flow alteration. Finally, amnesia might be caused by functional disturbances of other than the mesiotemporal brain regions-for example, the thalamus, ${ }^{18}$ which comprise a different venous drainage pattern.

Regarding the second precondition of the venous congestion hypothesis, the triggering VM, only $36 \%$ of patients in our study population fulfilled this criterion. However, this percentage might be an underestimation as it mainly relies on statements of the patients, which could have suffered from a partial retrograde amnesia. In other reports the proportion of unusual TGA precipitating activities ranges from $30-81 \% .{ }^{14810}$ However, some authors also included factors like 'emotional stress', ${ }^{10}$ which, in our opinion, do not reliably imply a likely VM. As all of the reported patient populations contain a proportion of 'spontaneous' TGAs the relevance of an initiating VM alone has still to be questioned.

In conclusion, the present study confirms the finding that TGA patients have a significantly higher prevalence of IJVVI. However, only approximately one third of them had a reliable history of a precipitating VM. We could not find any IJVVI related morphological intracranial venous drainage patterns on non-contrast venous MRA. Both findings argue against a simple causal relation between IJVVI and TGA. If VM and venous reflux do play a role within the aetiology of TGA, we assume that this probably occurs as cofactors of a still not understood multifactorial triggering mechanism, which could explain why reoccurrence rates of the condition are so low.

Research efforts to further analyse the relation between VM, IJVVI, and TGAs should be made towards an even more precise-for example, contrast-enhanced analysis-of the cerebral venous anatomy, including other venous drainage pathways like the vertebral and paraspinal veins in combination with a functional brain perfusion assessment. Assuming a multifactorial triggering mechanism, other coexisting contributing factors should be considered.

\section{Authors' affiliations}

S J Schreiber, F Doepp, J M Valdueza, Department of Neurology, University Hospital Charité, Berlin, Germany

R Klingebiel, Neuroradiology Section of the Department of Radiology, University Hospital Charité, Berlin, Germany

Competing interests: this study was supported by a grant from the Schering Forschungsgesellschaft mbH, Berlin, Germany.

\section{REFERENCES}

1 Caplan LR. Transient global amnesia. In: Vinken PJ, Bruyn GW, Klawans HL, eds. Handbook of clinical neurology.Vol 45. Amsterdam: Elsevier Science Publishers B, V., 1985:205-18.

2 Frederiks JAM. Transient global amnesia: an amnestic TIA. In: Markowitsch HJ, ed. Transient global amnesia and related disorders. Toronto: Hofgrefe and Huber Publ, 1990:28-47.

3 Hodges JR, Warlow CP. Syndromes of transient amnesia: towards a classification. A study of 153 cases. J Neurol Neurosurg Psych 1990:53:834-43.

4 Rösler A, Mras GJ, Frese A, et al. Precipitating factors of transient global amnesia. J Neurol 1999;246:53-4.

5 Schamschula RG, Soo MY. Transient global amnesia following cerebral angiography with non-ionic contrast medium. Australas Radio 1994;38:196-8.

6 Pantoni L, Lamassa M, Inzitari D. Transient global amnesia: a review emphasizing pathogenic aspects. Acta Neurol Scand 2000;102:275-83.

7 Lewis SL. Aetiology of transient global amnesia. Lancet 1998;352:397-9.

8 Sander D, Winbeck K, Etgen T, et al. Disturbance of venous flow patterns in patients with transient global amnesia. Lancet 2000;356:1982-4.

9 Akkawi NM, Agosti C, Rozzini L, et al. Transient global amnesia and disturbance of venous flow patterns. Lancet 2001;357:957.

10 Akkawi NM, Agosti C, Anzola GP, et al. Transient global amnesia: a clinical and sonographic study. Eur Neurol 2003;49:67-71.

11 Lepori D, Capasso P, Fournier D, et al. High-resolution ultrasound evaluation of internal jugular venous valves. Eur Radiol 1999;9:1222-6.

12 Lobato EB, Sulek CA, Moody RL, et al. Cross-sectional area of the right and left internal jugular veins. J Cardiothorac Vasc Anesth 1999;13:136-8.

13 Lichtenstein D, Saifi R, Augarde R, et al. The internal jugular veins are asymmetric. Usefulness of ultrasound before catheterization. Intensive Care Med 2001;27:301-5.

14 Hacker H. Normal supratentorial veins and dural sinuses. In: Newton TH, Potts DG, eds. Radiology of the skull and brain. Angiography. Saint Lovis: Mosby, 1974:1851-77. 
15 Kalbag RM. Anatomy and embryology of the cerebral venous system. In: Vinken PJ, Bruyn GW, eds. Handbook of clinical neurology. Vol 11 Amsterdam: North-Holland Publishing Company, 1972:45-64.

16 Bianchi D, Maeder P, Bogousslavsky J, et al. Diagnosis of cerebral venous thrombosis with routine magnetic resonance: an update. Eur Neurol 1998;40:179-90.
17 Ayanzen RH, Bird CR, Keller PJ, et al. Cerebral MR venography: normal anatomy and potential diagnostic piffalls. Am J Neuroradiol 2000;21:74-8.

18 Benabdelilil M, El Alaoui Faris M, Kissani N, et al. Neuropsychological disorders after bithalamic infarct caused by deep venous thrombosis. Rev Neurol (Paris) 2001;157:62-7.

\section{HISTORICAL NOTE}

\section{A note on scrivener's palsy}

$\mathrm{F}$ or many years-before its recognition as a focal dystonia-writers' cramp (scrivener's palsy (from the Latin scribere: to write)) was regarded as an occupational hazard akin to repetitive strain. Lucire ${ }^{1}$ in a provocative book suggests that the only new aspect of repetitive strain injury (RSI) is its name.

The first epidemics of writers' cramp were reported in the 1830s among clerks of the British Civil Service, where it was attributed to the new steel pen nib. Sir Charles Bell gave a description of these disorders:

"I have found the action necessary for writing gone, or the motions so irregular as to as make the letters be written zig-zag, whilst the power of strongly moving the arm for fencing, remained...

"The nerves and muscles are capable of their proper functions and proper adjustments; the defect is in the imperfect exercise of the will, or in the secondary influence the brain has over the relations established in the body. ${ }^{\prime 2}$

Duchenne reported:

"Functional spasm may occur anywhere, or affect a great number of voluntary and instinctive movements. Spasm of certain muscles of the hand which has been caused by abuse of writing, ... and which shows itself in the exercise of that function, first received in Germany the name of "Schreibekrampf," ... In France, it is called "crampe des écrivains."."3

Tenosynovitis was suggested as the cause in 1840 by GF Strohemeyer (1804-1876), whose device of cutting and separating tendons was a failure.

Samuel Solly (1805-1871) first used the name scrivener's palsy, which he reported in 1864, in a clinical lecture. ${ }^{4}$

He warned fellow surgeons:

"The paralyzed scrivener, though he cannot write, can amuse himself in his garden, can shoot, and cut his meat at the dinner table, indeed he can do almost anything he likes, except earn his daily bread as a scribbler... Upon your early correct diagnosis may depend the health and happiness of your subject. If you mistake its real nature and regard it as a sign of incipient softening of the brain, a mistake I have known to occur, you may destroy the happiness of your patient ..."

In 1870, Julius Althaus wrote: On scrivener's palsy and its treatment. Several $19^{\text {th }}$ century monographs described other occupational neuroses. Gowers regarded it as an:

"Occupation Neurosis: a convenient designation for a group of maladies in which certain symptoms are excited by the attempt to perform some often-repeated muscular action, commonly one that is involved in the occupation of the sufferer ...the pen does not move quite as intended....a slight involuntary movement causes an unintended mark ....now and then there is a distinct spasm, which cannot be controlled.... a disease easily imagined by those who have witnessed the disorder".

George M Beard who invented the term "neurasthenia" described 125 such cases. ${ }^{5}$ In the early 20th century, telegraphists in the UK began experiencing "telegraphist's cramp", thought to be caused by the rapid, repetitive movements required to send Morse code, affecting up to 18 per cent of British telegraphists. The suspicion was of a poor constitution, neurosis, and neurasthenic temperament.

\section{Samuel Solly (1805-1871)}

Born in London, the son of Isaac Solly and Mary Harrison, Samuel Solly was christened on 13 May 1805 at St Mary Axe. He was educated at Higham Hill Northampton (Disraeli's school). Solly was apprenticed to Benjamin Travers, a surgeon of St Thomas' Hospital, and graduated MRCS in 1828. He started in practice at St Mary Axe in 1830.

From 1833-9 he was a lecturer in anatomy and physiology at St Thomas' Hospital. In 1836 he was elected a Fellow of the Royal Society. He set up practice in 1840 in Savile Row and in 1853 was appointed as surgeon and lecturer at St Thomas', where he continued until illness forced resignation. He obtained the fellowship in 1843 and later became a council member and examiner at the Royal College of Surgeons of England. His publications included: The Human Brain, its configuration, structure, development, and physiology (London, 1836) -illustrated by references to the nervous system in the lower order of animals; Surgical Experiences (London, 1865)the substance of clinical lectures; and several of his watercolour pictures were hung at the Royal Academy. A marble bust was presented to St Thomas' Hospital and the "Solly Prize and Medal" was established in its Medical School in his memory.

He died on 21 September 1871 having fathered 11 children including Richard Harrison Solly who became a Professor of Botany, and Samuel Edwin Solly MD—a chest physician in Colorado Springs where he lived from 1900 having journeyed from England on account of chronic phthisis.

J M S Pearce 304 Beverley Road, Analby, Hull HU10 7BG, UK; jmspearce@freenet.co.uk

\section{References}

1 Lucire L. RSI, Belief and Desire. Univ New South Wales, 2003.

2 Bell C. Partial Paralyses of the Muscles of the Extremities'. The nervous system of the human body. London: Taylor and Francis, 1833:57-8.

3 Duchenne GBA. Poore GV, editor. Selections from the clinical works. London: New Sydenham Society; 1883, 399-409 from De L'electralisation localisée $3^{\text {rd }}$ edn. Paris: JB Baillière, 1855:1021-34.

4 Solly, Samuel (1805-1871) King's College London College Archives Papers of Samuel Solly, comprising surgical casebook containing notes on patients examined by him at St Thomas's Hospital, privately, and at Hanwell.

5 Beard GM. A Practical treatise on nervous exhaustion (neurasthenia): its symptoms, nature, sequences, treatment. reprinted special edn. Birmingham, Al: Classics of Psychiatry \& Behavioral Sciences Library, 1991. 